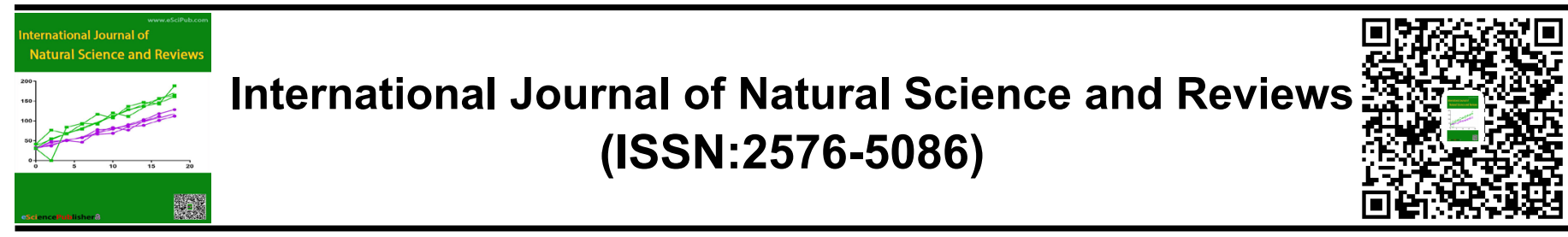

\title{
Motor Vehicle Maintenance Scheduling Model Based on Genetic algorithm
}

Yishuai Tian ${ }^{1 *}$, Mingxuan Lou', Xinhai Zhao', Yalong Zheng ${ }^{1}$

${ }^{1}$ College of Hydraulic \& Environmental Engineering, China Three Gorges University, Yichang 443002, China.
ABSTRACT
To solve the problem of EMU maintenance planning time, we established a hybrid linear programming model based on adaptive genetic algorithm. According to the different levels of bullet train maintenance and the different arrival time, the constraint conditions are found, and the overall minimum maintenance time is taken as the objective function. Then the *Correspondence to Author: problem is transformed into a hybrid Flow-shop scheduling problem with unique process constraints, and an adaptive genetic algorithm is used to solve it again. It is concluded that How to cite this article:
the total maintenance takes 1125 minutes, and no blockage Yishuai Tian, Mingxuan Lou, occurred in all EMU during the maintenance period. To provide a Xinhai Zhao, Yalong Zheng. Motor consistent basis for bullet train maintenance time and scheduling.
College of Hydraulic \& Environmen- tal Engineering, China Three Gprg- es University, Yichang 443002, China. Keywords: Linear programmin
Flow-shop; Train maintenance Model Based on Genetic algorithm. International Journ-al of Natural Science and Reviews, 2020; 5:13

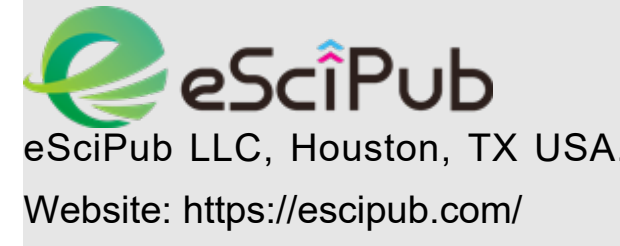




\section{Introduction}

Full and high efficiency is the fundamental requirement of high-speed railway EMU operation [1]. EMU maintenance is an essential means to ensure the safe and efficient service of EMU. The optimization of the EMU maintenance plan is of great significance to improve the EMU operation efficiency and reduce the EMU maintenance cost. at the same time, it can provide a decision-making basis for the optimal allocation of maintenance capacity and the number of spare cars. The place responsible for the maintenance and maintenance of the bullet train is called the EMU operation group, and 50 EMU have been built in China ${ }^{[2]}$. the support of EMU is divided into different maintenance levels, different grades correspond to different processes, and each method of different types of EMU takes different time ${ }^{[1-3]}$.

To dispatch the incoming train, to minimize the time required. For this reason, this paper estab- lished a train maintenance scheduling model based on a genetic algorithm, obtained the objective function and constraint conditions according to the requirements, and used a genetic algorithm to solve it to provide a relevant basis for bullet train maintenance time and scheduling $[4,5]$.

\section{Problems to be solved}

According to the running time, course and maintenance cycle of the train, the maintenance of EMU is divided into different maintenance levels, and various maintenance levels correspond to different process combinations, as shown in Table 1. There is 1 workshop in the process a, b and $\mathrm{c}, 3$ workshops in process $\mathrm{d}$ and 2 workshops in e, and time consumption is the same in different workshops in the same process. The time required for each operation of different types of motor cars is given. The total time required to overhaul these trains is calculated according to the information of the trains arrived ${ }^{[6]}$.

Table 1 Arrival information of motor cars, types of maintenance and combination of working procedures

\begin{tabular}{cccc}
\hline Arrival time & Type of motor car & Maintenance type & Corresponding process combination \\
\hline $0: 16$ & $\mathrm{CRH} 2$ & IV & $\mathrm{a} \rightarrow \mathrm{c} \rightarrow \mathrm{d} \rightarrow \mathrm{e}$ \\
$0: 47$ & $\mathrm{CRH} 5$ & II & $\mathrm{a} \rightarrow \mathrm{b} \rightarrow \mathrm{c}$ \\
$1: 22$ & $\mathrm{CRH} 2$ & I & $\mathrm{a} \rightarrow \mathrm{b} \rightarrow \mathrm{c}$ \\
$2: 00$ & $\mathrm{CRH} 6$ & $\mathrm{I}$ & $\mathrm{a} \rightarrow \mathrm{b}$ \\
$2: 20$ & $\mathrm{CRH} 3$ & II & $\mathrm{a} \rightarrow \mathrm{b} \rightarrow \mathrm{d}$ \\
$3: 05$ & $\mathrm{CRH} 6$ & I & $\mathrm{a} \rightarrow \mathrm{b} \rightarrow \mathrm{c}$ \\
$3: 31$ & $\mathrm{CRH} 2$ & $\mathrm{~V}$ & $\mathrm{a} \rightarrow \mathrm{b} \rightarrow \mathrm{c} \rightarrow \mathrm{d} \rightarrow \mathrm{e}$ \\
\hline
\end{tabular}

Table 2 Time required for various types of motor cars in different processes

\begin{tabular}{cccccc}
\hline \multirow{2}{*}{$\begin{array}{c}\text { Type of mo- } \\
\text { tor car }\end{array}$} & $\mathrm{a}$ & $\mathrm{b}$ & $\mathrm{c}$ & $\mathrm{d}$ & $\mathrm{e}$ \\
\cline { 2 - 6 } & 1 & 2 & 1.5 & 4 & 7 \\
$\mathrm{CRH} 2$ & 0.8 & 2.4 & 0.5 & 4.8 & 6.5 \\
$\mathrm{CRH} 3$ & 2.5 & 1.5 & 3 & 6 \\
$\mathrm{CRH} 5$ & 1.3 & 2.7 & 0.3 & 5 & 7 \\
CRH6 & 1 & & &
\end{tabular}

3. Establishment of mixed Linear programming Model

\subsection{Determination of objective function}

Let $o, p, q$, be the number of workshops in the three processes of, $a, b, c$, among which $o=1,2,3 . p=1,2, \cdots, 8 . q=1,2, \cdots, 5$

$r=1,2,3, s=1,2$. Let $m, n$ is the serial number of the motor cars driven into the overhaul and the number of categories in the maintenance workshop, among which 
$m=1,2, \cdots, 7, n=1,2, \cdots, 5$. To improve the overall maintenance efficiency of the incoming maintenance EMU of different types of bullet trains at different levels of maintenance, the objective function is determined as the shortest maintena-

$$
\min \max _{1 \leq m \leq 7}\left\{\max _{1 \leq n \leq 5}\left\{T_{m n}\right\}\right\}
$$

\subsection{Establishment of constraints}

Establishment of bullet train type 0-1 matrix $K_{r \times m}$ coach. $K_{r \times m}$ means that the m-th EMU is an r-type bullet train. $r=1,2,3,4$ denotes the model of each type of motor car, for example,

$$
K_{r \times m}=\left[\begin{array}{lll}
K_{11} & \cdots & K_{1 r}
\end{array}\right]_{r \times m}
$$

$(r=1,2, \cdots, 4, m=1,2, \cdots, 7)$

Establishment of $L_{n \times r}$ repair data Matrix for various types of Motor cars. Let $L_{n r}$ denote the processing time of the r-type motor car in the nth

$$
L_{n \times r}=\left[\begin{array}{lll}
L_{11} & \cdots & L_{1 r}
\end{array}\right]_{n \times r}
$$

$(n=1,2, \cdots 5, r=1,2, \cdots, 4)$

The establishment of the relation matrix $E_{n \times e}$ repair between maintenance type and workshop 01Since motor cars with different maintenance levels enter different workshops in a different order, in which $e=1,2,3,4,5$ denotes the

$$
E_{n \times e}=\left[\begin{array}{lll}
E_{11} & \cdots & E_{1 e}
\end{array}\right]_{n \times e}
$$

Where $E_{n e}=1$ means that workshop $\mathrm{n}$ will be used in the maintenance of level e, $E_{n e}=0$ which means that workshop $\mathrm{n}$ will not be used in the maintenance of level $e$.

Establishment of vehicle serial number and maintenance 0 to 1 type matrix $F_{e \times m}$ repair. Because the bullet trains entering the station at different times are not only different in time and

$$
F_{e \times m}=\left[\begin{array}{lll}
F_{11} & \cdots & F_{1 m}
\end{array}\right]_{e \times m}
$$$$
(e=1,2, \cdots, 5, m=1,2, \cdots, 7)
$$

The establishment of a time constraint $S_{m \times n}$ matrix. Because the time of the bullet train entering the inspection and repair station is not only different, its maintenance grade is different, and the maintenance time in each workshop is

$$
S_{m \times n}=\left[\left(E_{n \times e} \cdot F_{e \times m}\right) \times\left(K_{r \times m} \cdot L_{n \times r}\right)\right]_{m \times n}(\forall n, \forall r, \forall m, \forall e)
$$

Establishment of constraints for entering the maintenance process. Let $t_{m}\left(t_{1}, t_{2}, \cdots t_{m}, \cdots t_{7}\right)$ denote the time point at which the m-th EMU enters the maintenance station, because it may 
need to queue up before entering the mainte- established as follows: nance station. Therefore, the constraints can be

$$
t_{m} \leq t_{m a} \quad(m=1,2, \cdots, 7)
$$

Whether the bullet train has entered the inspec- the j-th workshop of the I-th process, and when tion station. Let $A_{m}^{i j}$ denote whether the $\mathrm{m}$-th car $\quad A_{m}^{i j}=1$ indicates that the $\mathrm{m}$-th car will enter the enters the j-th workshop of the I-th process, j-th workshop of the I-th process. Get the followwhere means that the $\mathrm{m}$-th car does not enter ing constraints:

$$
A_{m}^{i j}=\{0,1\}
$$

Whether the bullet train occupies the inspection station. Let the value of $B_{m}^{i j}$ indicating whether the m-th car T occupies the j-th workshop of the I-th process at any time. Get the constraint condition $B_{m}^{i j}=\{0,1\}$, When means that the $\mathrm{m}$-th car $T$ does not occupy the j-th workshop of the I-th process at any time; When $B_{k}^{i j}=1$ means that the

$$
B(T)_{m}^{i j}=\left\{\begin{array}{ll}
0 & T<t_{m n} \\
1 & t_{m n} \leq T \leq T_{m n} \\
0 & T>T_{m n}
\end{array} \quad(m=1,2, \cdots, 7, n=1,2, \cdots, 5)\right.
$$

Restrictions on the number of motor cars in the workshop. For $T$ at any time, there will be at most one set of bullet trains in each workshop in the m-th car occupies the j-th workshop of the i-th process at any time. According to the actual situation, there is $B_{m}^{i j}=0$ when the train has not entered or has been overhauled. $t_{i j} \mathrm{~T}$ denotes the entry time of $m E M U$ in the nth maintenance workshop, $T$ represents any time, $T \in(0,+\infty)$.So the $B_{m}^{i j}$ constraint is as follows:

three types of maintenance procedures of $a, b, c, d, e$, so the constraints can be established as follows:

$$
\sum_{m} B(T)_{m}^{i j} \leq 1 \quad(T \in(0,+\infty), i=1,2, \cdots, 5, \forall j)
$$

The constraint of time cohesion. According to the requirements of the topic, the switching time between EMU is ignored. For any group of EMU, the end time of $m$ in the previous workshop is the entry time of entering the next workshop. Therefore, the constraints are established as follows:

$$
T_{m(n+1)}=t_{m n} \quad(m=1,2, \cdots, 7, n=1,2,3,4)
$$

The restriction of EMU entering the station. Any EMU m can only enter one workshop after each maintenance process, and some workshops caused by different maintenance levels do not

$$
\sum_{i} A_{m}^{i j}=1 \quad(m=1,2, \cdots, 7, \forall j)
$$

Maintenance time constraint. $S_{m n}$ indicates the processing time of the $m$-th EMU in process $n$. As different types of motor cars spend different time in different workshops in the same process, and different maintenance workshops with different maintenance levels, if the workshop in the

$$
T_{m n}-t_{m n} \geq S_{m n} \quad(m=1,2, \cdots, 7, n=1,2, \cdots, 5)
$$

To sum up, the single-objective nonlinear progr- amming model is established as follows: next process is full, we need to wait in the original workshop. For any group of motor cars, it can be found that the stay time in different processes may exceed the maintenance time. Therefore, constraints can be established as follows: 


$$
\text { s.t. }\left\{\begin{array}{l}
\min \max _{1 \leq m \leq 7}\left\{\max _{1 \leq n \leq 5}\left\{T_{m n}\right\}\right\} \\
S_{m \times n}=\left[\left(E_{n \times e} \cdot F_{e \times m}\right) \times\left(K_{r \times m} \cdot L_{n \times r}\right)\right]_{m \times n} \\
t_{m} \leq t_{m a} \quad(m=1,2, \cdots, 7) \\
A_{m}^{i j}=\left\{\begin{array}{l}
0,1\} \\
B(T)_{m}^{i j}=\left\{\begin{array}{ll}
0 & T<t_{m n} \\
1 & t_{m n} \leq T \leq T_{m n} \\
0 & T>T_{m n}
\end{array} \quad(m=1,2, \cdots, 7, n=1,2, \cdots, 5)\right. \\
\sum_{m} B(T)_{m}^{i j} \leq 1 \quad(T \in(0,+\infty)) \\
T_{m n}=t_{m(n+1)} \\
\sum_{i} A_{m}^{i j}=1 \\
T_{m n}-t_{m n} \geq S_{m n}
\end{array}\right.
\end{array}\right.
$$

3.3 Application of Genetic Algorithm in Cal- and genetic algorithm are all algorithms to solve culation of Motor Vehicle Maintenance Time At present, this kind of NP problem is mainly solved by high-end algorithms ${ }^{[8]}$. An intelligent algorithm is needed to solve the problem. The this problem. Among them, the genetic algorithm is widely used. Therefore, a genetic algorithm is used as a method to solve the problem. The flow neural network, simulated annealing algorithm,

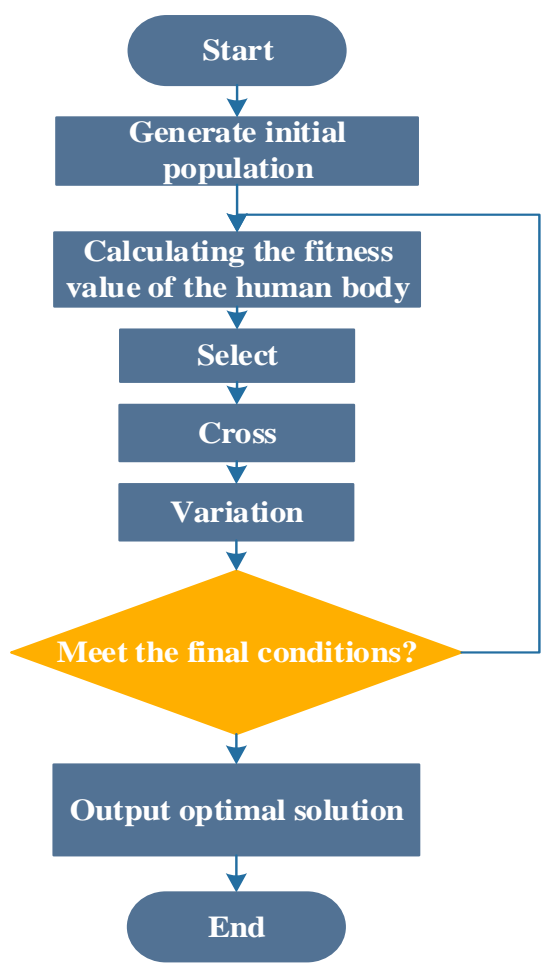

Fig. 1 Flow chart of the genetic algorithm

\section{Results and result analysis}

Based on the MATLAB platform, input the processing time and processing level of different types and different processes. The population size is $\mathrm{N}=50$, the mutation probability is $\mathrm{P}=0.1$, and the number of iterations is 1000 . Five iterations are carried out by changing the number of iterations to solve the minimum completion time. The specific simulation results are as follows 


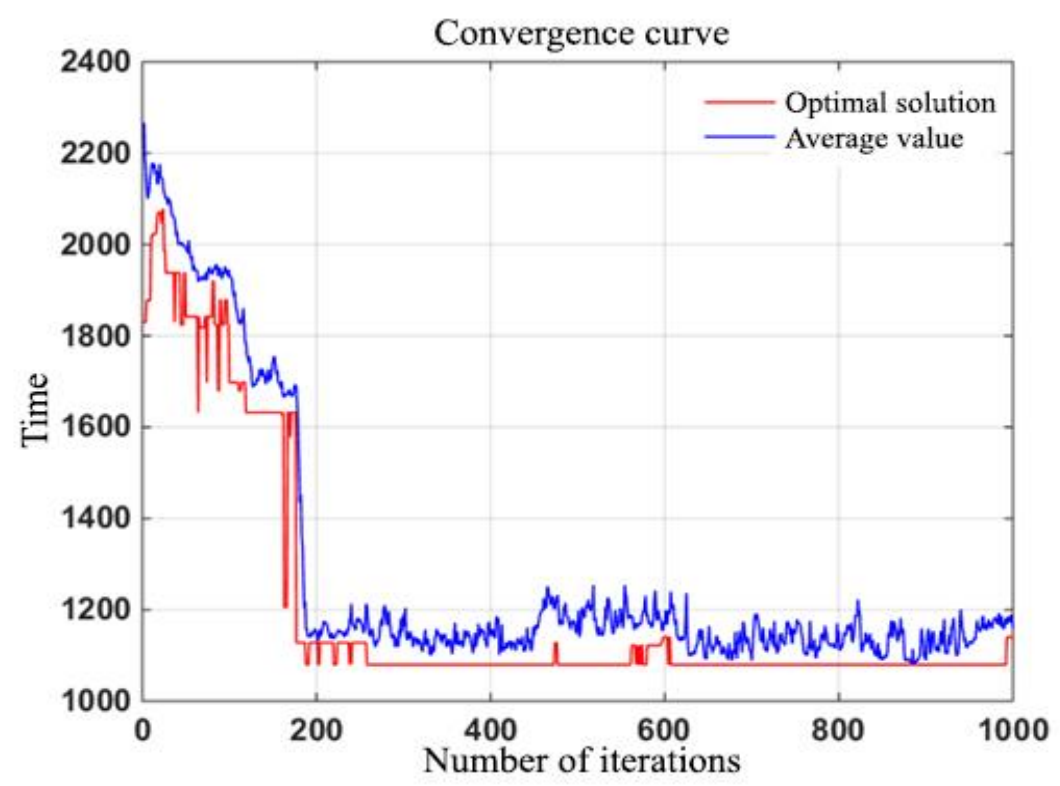

Fig. 2 Convergence result of genetic algorithm

As shown in Fig. 2, when the solution obtained by the number of iterations is the optimal solution. The last one leaves at 7:01 pm, and the total maintenance time is 1125 minutes. The Gantt chart was drawn using the genetic algorithm, as shown in Fig. 3.

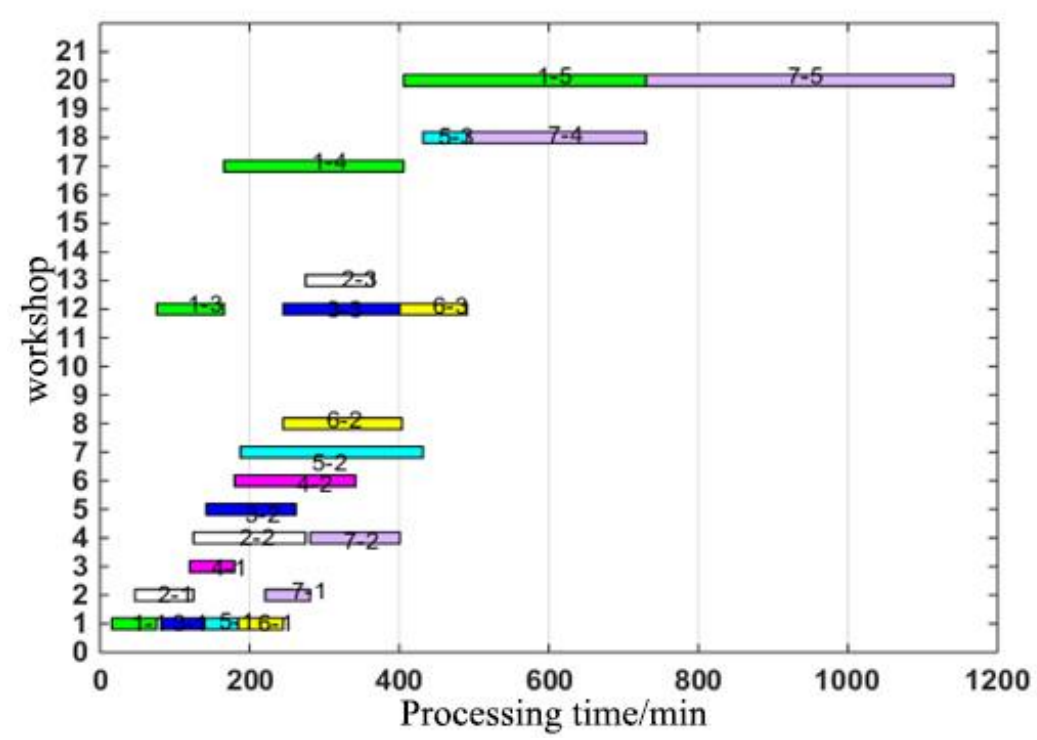

Fig. 3 Gantt chart of motor car maintenance time

As shown in Fig.3, the rectangles of seven different colors represent seven different EMU. The process of the motor car is explained by tagging $m-n$, where $m$ represents the serial number of the motor car, and $\mathrm{n}$ represents the serial number of the process. Numbers represent the sequence of processes. The $y$-axis represents the 21 workshop serial numbers of 5 processes. The length of the color block in the Gantt chart represents the stay time of all types of motor cars in each process. The solution to the F-TSP problem under the genetic algorithm needs to constantly change the number of iterations to find a globally optimal solution.

\section{Conclusion}

The adaptive genetic algorithm model and the hybrid linear programming model are established. According to the different maintenance levels and arrival time, the minimum maintenance time is taken as the objective function, and the problem is transformed into a hybrid 
Flow-shop scheduling problem with special process constraints, and the adaptive genetic algorithm is used to solve it again. It is concluded that the total maintenance takes 1125 minutes, and no blockage occurred in all EMU during the maintenance period. To provide a consistent basis for bullet train maintenance time and scheduling.

\section{References}

1. Fei Gao, Yu Pan. Beijing EMU maintenance base and EMU maintenance[J]. Railway rolling stock, 2010,30 (04): 77-81.

2. Meihua Zhu. Research on optimization of the use of EMU under passenger dedicated line[D]. Lanzhou Jiaotong University, 2013.

3. Jian Li. Research on the comprehensive optimization method of EMU operation and maintenance plan [D]. Beijing Jiaotong University, 2017.

4. Maroti G, Kroon L. Maintenance routing for train units: The interchange model[J]. COMPUTERS \& OPERATIONS RESEARCH,2007,34(4):11211140.

5. Zhang D, Hu H, Liu Y, et al. Railway Train Wheel Maintenance Model and Its Application[J]. TRANSPORTATION RESEARCH RECORD, 2014(2448):28-36.

6. Hani $Y$, Amodeo L, Yalaoui F, et al. Simulation based optimization of a train maintenance facility[J]. JOURNAL OF INTELLIGENT MANUFACTURING, 2008,19(3):293-300.

7. Reich D. A linear programming approach for linear programs with probabilistic constraints[J]. EUROPEAN JOURNAL OF OPERATIONAL RESEARCH, 2013,230(3):487-494.

8. Ding C, Chen L, Zhong B. Exploration of intelligent computing based on improved hybrid genetic algorithm[J]. CLUSTER COMPUTINGTHE JOURNAL OF NETWORKS SOFTWARE TOOLS AND APPLICATIONS, 2019,224:S9037 $-\mathrm{S} 9045$ 